\title{
Brain hypothermia therapy for childhood acute encephalopathy based on clinical evidence (Review)
}

\author{
GEORGE IMATAKA and OSAMU ARISAKA \\ Department of Pediatrics, Dokkyo Medical University School of Medicine, Tochigi 321-0293, Japan
}

Received July 31, 2015; Accepted September 15, 2015

DOI: $10.3892 /$ etm.2015.2760

\begin{abstract}
Although previous studies have reported on the effectiveness of brain hypothermia therapy in childhood acute encephalopathy, additional studies in this field are necessary. In this review, we discussed brain hypothermia therapy methods for two clinical conditions for which sufficient evidences are currently available in the literature. The first condition is known as hypoxic-ischemic encephalopathy and occurs in newborns and the second condition is acute encephalopathy which occurs in adults following cardiopulmonary resuscitation associated with out-of-hospital cardiac arrest state resulting from ventricular arrhythmia. Furthermore, we assessed the prospects of applying these therapies to acute encephalopathy in children.
\end{abstract}

\section{Contents}

1. Clinical evidence for brain hypothermia therapy

2. Treatment for brain hypothermia therapy of childhood with the onset of acute encephalopathy

3. Conclusion

\section{Clinical evidence for brain hypothermia therapy}

Clinical reports have focused on the effectiveness of brain hypothermia therapy for acute encephalopathy in children. However, there is a lack of high-quality studies with a large number of cases that can show the effectiveness and safety of clinical methods used in brain hypothermia therapy in childhood acute encephalopathy. There are currently two methods of therapy for which numerous high-quality reports are available in the literature. These studies mostly focused on

Correspondence to: Dr George Imataka, Department of Pediatrics, Dokkyo Medical University School of Medicine, 880 Kitakobayashi, Mibu, Shimotsuga, Tochigi 321-0293, Japan

E-mail: geo@dokkyomed.ac.jp

Key words: hypothermic therapy, child, status epilepticus, Targeted temperature management, cooling brain hypothermia therapy and reported on hypoxic-ischemic encephalopathy in newborns and acute encephalopathy in adults following cardiopulmonary resuscitation associated with out-of-hospital cardiac arrest state resulting from ventricular arrhythmia.

Six large-scale clinical studies focusing on hypoxic-ischemic encephalopathy of newborns were reported during the period 2005-2010. The studies were conducted mainly in western countries, China, and Oceania. One of these clinical studies was the TOBY trial which was conducted in 2009 and included 163 cases (1). Investigators in the TOBY trial performed body cooling for $72 \mathrm{~h}$ and reduced the temperature (rectal temperature) to $33^{\circ} \mathrm{C}$ and $34^{\circ} \mathrm{C}$. The results revealed a significant reduction in mortaligy or severe after-effects after 18 months from the treatment (1). Follow-up studies conducted during the childhood period indicated improvements in the cognitive ability of the children involved (2).

In a report conducted on 100 cases in 2010 in China, the nasopharynx was cooled for $72 \mathrm{~h}$ at $34 \pm 0.2^{\circ}$ using a selective head cooling method (3). Results from that study showed a significant difference in mortality and severe after effects after 18 months (3). As a result, the brain hypothermia therapy was recommended for childhood hypoxic-ischemic encephalopathy by the International Liaison Committee on Resuscitation in 2010 (4).

Concerning the out-of-hospital cardiopulmonary arrest state in adults, two high-quality reports were published in 2002. In one report, 275 patients were resuscitated from the cardiopulmonary arrest state. In that study 137 patients were assigned to the hypothermia group and 138 to the normothermia group (5). Th difference in mortality rates after 6 months was compared between the two groups. The mortality rates 6 months after cardiac arrest was 14 percentage points lower in the hypothermia group than that in the normothermia group, while $55 \%$ of patients in the hypothermia group had a favorable neurologic outcome, as compared with $39 \%$ in the normothermia group. Authors of that study concluded that the systemic cooling to a bladder temperature between $32^{\circ} \mathrm{C}$ and $34^{\circ} \mathrm{C}$ for $24 \mathrm{~h}$ increased the chance of survival and produced a favorable neurologic outcome (5).

In the second study, 77 patients were assigned to treatment with hypothermia or normothermia (6). Core body temperature of patients in the hypothermia group was reduced to $33^{\circ} \mathrm{C}$ within $2 \mathrm{~h}$ after the return of spontaneous circulation and maintained at that temperature for $12 \mathrm{~h}$. Results from 
that study revealed that the life prognosis improved in the hypothermia group (6). Authors of that study concluded that induced hypothermia improved outcomes in comatose patients after resuscitation from out-of-hospital cardiac arrest (6). Consequently, in 2002, the American Heart Association and the European Resuscitation Council recommended the brain hypothermia therapy as a viable treatment following resuscitation from cardiopulmonary arrest state in adults.

However, few studies have reported brain hypothermia therapy for childhood acute encephalopathy. Studies conducted on the effectiveness of brain hypothermia therapy for childhood acute encephalopathy exist only at the case report level, and a large-scale clinical research report has yet to be performed. In one clinical study reporting on brain hypothermia therapy in 38 children, caused by out-of-hospital cardiac arrest, the clinical effects were not confirmed (7). Acute encephalopathy is an aspect of this disease with a broad spectrum of symptoms including a variety of pathological conditions (such as fever, infection, epilepsy, immunological abnormalities, metabolic issues, hypoxia and genetic factors) caused by brain dysfunction. In childhood acute encephalopathy, therefore, it is preferable to select a treatment method according to its class and the severity of the complication.

In another case report on brain hypothermia therapy the authors reported about a noteworthy outcome in an infant with epilepticus-type acute encephalopathy who suffered from cardiac arrest and cardiopulmonary resuscitation (8). The results reported in that study were very encouraging and may lead to more discussions and even initiate additional studies in future.

\section{Treatment for brain hypothermia therapy of childhood with the onset of acute encephalopathy}

The method of brain hypothermia therapy for children has yet to be widely introduced as a viable type of treatment. There is only one protocol report on the method of brain hypothermia therapy for children (9). This protocol report was developed in a single facility, without establishing any standard treatment method or safety precautions. Therapy using various drugs, such as pulse steroids, high-dose $\gamma$-globulin and anticonvulsants was used in combination for brain hypothermia (9); however, no study group has conducted a comparative study on the clinical effects of these medications.

The specifics of brain hypothermia therapy in childhood acute encephalopathy remain to be determiend. For example, we do not have a complete understanding regarding the most efficient temperature or the most effective treatment period duration that should be used for patients such as children.

In a multi-facility cooperative study conducted in Europe and Australia, a study on 950 cases of adults suffering from acute brain disorder following cardiac arrest was conducted in order to compare their prognosis after 6 months (10). The targeted control body temperatures were divided into two groups with a temperature of $33^{\circ} \mathrm{C}$ and $36^{\circ} \mathrm{C}$. The data obtained from these groups were compared. Results of that study reported no substantial amelioration in the prognosis in the groups investigated (10).
Based on the results obtained in the abovementioned study, the brain normal-temperature method or targeted temperature management (TTM) has been recently attempted as a practical treatment method for childhood acute encephalopathy. Brain normal-temperature method or TTM were also employed as treatment methods for maintaining the brain or body temperature at $36^{\circ}$ (11). Currently, however, this procedure remains at the investigation stage, while there is no evidence indicating the obvious effectiveness of TTM.

When applying brain hypothermia therapy to childhood acute encephalopathy, the timing and safety from the onset of acute encephalopathy until brain hypothermia therapy commences should be also studied satisfactorily. During the early phase of the childhood acute encephalopathy, the damage to the brain is more severe. This damage increases the risk of secondary disorders such as delayed energy production and defective energy utilization, neuronal edema, and cerebral blood flow perfusion abnormality (9). Therefore, the therapeutic time window (TTW) is limited and there is only a narrow time window to treat severe acute encephalopathy effectively (10). A study on TTW was conducted in 10 Japanese facilities equipped with a pediatric intensive care unit (PICU) (12). The Japanese study group worked on 43 cases of which 27 were submitted to mild hypothermia and 16 cases to normothermia.

Brain hypothermia therapy was conducted on children with acute encephalitis and acute encephalopathy. Children were subjected to the brain hypothermia therapy for $48 \mathrm{~h}$ using the whole body cooling system to set the body temperature at $33.5-35^{\circ} \mathrm{C}(12)$. Results from that study demonstrated that brain hypothermia therapy in the early stage of the disease significantly improves the neurological prognosis (12).

A contradictory report offer evidence on the poor prognosis of brain hypothermia therapy in the early stage of disease (13) making the selection of an appropriate therapeutic method a challenge. Brain hypothermia therapy in children is a treatment that should be performed in highly advanced medical facilities or intensive care rooms in order to provide optimal care and monitoring facilities.

In the brain hypothermia therapy whole-body management at the PICU level is required. In some cases of brain hypothermia therapy, steroid pulse therapy, $\gamma$-globulin therapy, and intravenous therapy of various anti-seizure drugs are often used together (9). Therefore, to confirm the clinical effects of this therapy, it is essential to study the clinical courses of treatment by dividing the cases into two groups: i) group being administered the brain hypothermia therapy alone and ii) group receiving the treatment using the concomitant drugs. The number of studies conducted on this subject are currently insufficient. Informed consent, however, remains a determining factor in studies on brain hypothermia therapy.

\section{Conclusion}

As there are many cases with good prognosis using medical therapy alone in childhood acute encephalopathy, overtreatment must be sufficiently taken into consideration when selecting brain hypothermia therapy. In future, in order to 
determine the effects and prognosis of the brain hypothermia therapy for acute encephalopathy with a broad spectrum of symptoms including the epilepticus-type encephalopathy in children, evidence worthy of the objective evaluation in accordance with the clinical symptoms should be obtained and assessed.

\section{References}

1. Azzopardi DV, Strohm B, Edwards AD, Dyet L, Halliday HL, Juszczak E, Kapellou O, Levene M, Marlow N, Porter E, et al; TOBY Study Group: Moderate hypothermia to treat perinatal asphyxial encephalopathy. N Engl J Med 361: 1349-1358, 2009.

2. Azzopardi D, Strohm B, Marlow N, Brocklehurst P, Deierl A, Eddama O, Goodwin J, Halliday HL, Juszczak E, Kapellou O, et al; TOBY Study Group: Effects of hypothermia for perinatal asphyxia on childhood outcomes. N Engl J Med 371: 140-149, 2014.

3. Zhou WH, Cheng GQ, Shao XM, Liu XZ, Shan RB, Zhuang DY, Zhou CL, Du LZ, Cao Y, Yang Q, Wang LS; China Study Group: Selective head cooling with mild systemic hypothermia after neonatal hypoxic-ischemic encephalopathy: A multicenter randomized controlled trial in China. J Pediatr 157: 367-372, 372.e1-3, 2010

4. Perlman JM, Wyllie J, Kattwinkel J, Atkins DL, Chameides L, Goldsmith JP, Guinsburg R, Hazinski MF, Morley C, Richmond S, et al; Neonatal Resuscitation Chapter Collaborators: Part 11: Neonatal resuscitation: 2010 International consensus on cardiopulmonary resuscitation and emergency cardiovascular care science with treatment recommendations. Circulation 122(16 Suppl 2): S516-S538, 2010

5. Hypothermia after Cardiac Arrest Study Group: Mild therapeutic hypothermia to improve the neurologic outcome after cardiac arrest. N Engl J Med 346: 549-556, 2002.
6. Bernard SA, Gray TW, Buist MD, Jones BM, Silvester W, Gutteridge G and Smith K: Treatment of comatose survivors of out-of-hospital cardiac arrest with induced hypothermia. N Engl J Med 346: 557-563, 2002.

7. Moler FW, Silverstein FS, Holubkov R, Slomine BS, Christensen JR, Nadkarni VM, Meert KL, Clark AE, Browning B, Pemberton VL, et al; THAPCA trial investigators: therapeutic hypothermia after out-of-hospital cardiac arrest in children. N Engl J Med 372: 1898-1908, 2015.

8. Imataka G, Tsuboi Y, Kano Y, Ogino K, Tsuchioka T, Ohnishi T, Kaji Y, Wake K, Ichikawa G, Suzumura H, et al: Treatment with mild brain hypothermia for cardiopulmonary resuscitation after myoclonic seizures in infant with robertsonian type of trisomy 13. Eur Rev Med Pharmacol Sci 19: 2852-2855, 2015.

9. Imataka G, Wake K, Yamanouchi H, Ono K and Arisaka O: Brain hypothermia therapy for status epilepticus in childhood. Eur Rev Med Pharmacol Sci 18: 1883-1888, 2014.

10. Nielsen N, Wetterslev J, Cronberg T, Erlinge D, Gasche Y, Hassager C, Horn J, Hovdenes J, Kjaergaard J, Kuiper M, et al; TTM Trial Investigators: Targeted temperature management at $33^{\circ} \mathrm{C}$ versus $36^{\circ} \mathrm{C}$ after cardiac arrest. N Engl J Med 369: 2197-2206, 2013.

11. Nishiyama M, Tanaka T, Fujita K, Maruyama A and Nagase H: Targeted temperature management of acute encephalopathy without AST elevation. Brain Dev 37: 328-333, 2015.

12. Kawano G, Iwata O, Iwata S, Kawano K, Obu K, Kuki I, Rinka H, Shiomi M, Yamanouchi H, Kakuma T, et al; Research Network for Acute Encephalopathy in Childhood: Determinants of outcomes following acute child encephalopathy and encephalitis: Pivotal effect of early and delayed cooling. Arch Dis Child 96: 936-941, 2011.

13. Imataka G, Wake K, Suzuki M, Yamanouchi H and Arisaka O: Acute encephalopathy associated with hemolytic uremic syndrome caused by Escherichia coli O157: H7 and rotavirus infection. Eur Rev Med Pharmacol Sci 19: 1842-1844, 2015. 